\title{
Active site alanine substitutions can convert deubiquitinating enzymes into avid ubiquitin-binding domains
}

Marie Morrow ${ }^{1}$, Michael Morgan ${ }^{1}$, Marcello Clerici ${ }^{2, \&}$, Katerina Growkova ${ }^{3}$, Ming Yan ${ }^{1}$, David Komander ${ }^{4}$, Michal Simicek ${ }^{3,4}$, Titia Sixma ${ }^{2}$ and Cynthia Wolberger ${ }^{1, *}$

${ }^{1}$ Dept. of Biophysics and Biophysical Chemistry, Johns Hopkins University School of Medicine, Baltimore, MD 21205 USA

${ }^{2}$ Division of Biochemistry and Oncode Institute, Netherlands Cancer Institute, Plesmanlaan 121, 1066 CX Amsterdam, The Netherlands

${ }^{3}$ Faculty of Medicine, University of Ostrava, Ostrava, Czech Republic.

${ }^{4}$ Medical Research Council Laboratory of Molecular Biology, Francis-Crick Avenue, Cambridge Biomedical Campus, Cambridge CB2 OQH, U.K.

${ }^{\&}$ Current address: Department of Biochemistry, University of Zurich, Winterthurerstrasse 190, CH-8057 Zurich, Switzerland

*Corresponding author: cwolberg@jhmi.edu 


\section{ABSTRACT}

A common strategy for studying the biological role of deubiquitinating enzymes (DUBs) in different pathways is to study the effects of replacing the wild type DUB with a catalytically inactive mutant in cells. We report here that a commonly studied DUB mutation, in which the catalytic cysteine is replaced with alanine, can dramatically increase the affinity of some DUBs for ubiquitin. Overexpression of these tight-binding mutants thus has the potential to sequester cellular pools of monoubiquitin and ubiquitin chains. As a result, cells expressing these mutants may display unpredictable dominant negative physiological effects that are not related to loss of DUB activity. The structure of the SAGA DUB module bound to free ubiquitin reveals the structural basis for the 30 fold higher affinity of $\mathrm{Ubp} 8{ }^{\mathrm{C} 146 \mathrm{~A}}$ for ubiquitin. We show that an alternative option, substituting the active site cysteine with arginine, can inactivate DUBs while also decreasing the affinity for ubiquitin. 


\section{Introduction}

Deubiquitinating enzymes (DUBs) play fundamental roles in ubiquitin signaling through their ability to remove ubiquitin from target proteins and disassemble polyubiquitin chains [1]. These enzymes cleave the isopeptide linkage between the C-terminus of ubiquitin and the substrate lysine or, in some cases, the peptide bond between ubiquitin and a substrate protein $\mathrm{N}$-terminus. The human genome encodes more than $90 \mathrm{DUBs}$, which can be grouped into families based on their fold: ubiquitin-specific protease (USP), ubiquitin carboxy-terminal hydrolase [2], ovarian tumor family (OTUs), Machado-Joseph domain (MJD) family, and JAMM/MPN domain (JAMMs), as well as the recently-discovered MINDY family [3-5]. Studies of deletions as well as diseasecausing mutations have revealed specific functions for individual DUBs in biological processes including proteasomal degradation, protein trafficking, transcription, DNA repair, infection and inflammation [6-8]. The involvement of DUBs in a variety of oncogenic, inflammation, and neurodegenerative pathways [9] has made these enzymes attractive targets for drug discovery $[10,11]$.

A common approach to determining the role of a particular DUB in cellular pathways is to knock down expression of the endogenous DUB and express a catalytically inactive version of the enzyme. With the exception of the JAMM domain family, which are metalloproteases, all other DUBs are cysteine proteases with a classical papain-like active site in which the cysteine is activated by an adjacent histidine [12]. Cysteine protease DUBs are typically inactivated by substituting the active site cysteine with another residue. Resulting changes in substrate ubiquitination or downstream signaling pathways in cells expressing the mutant DUB are generally assumed to be due to the absence of deubiquitinating activity, with the notable exceptions of OTUB1, which inhibits E2 enzymes by a mechanism independent of catalytic activity [13-15] and OTUD4, which serves as a scaffold for USP enzymes [16]. Whereas serine is the most conservative substitution for the active site cysteine, alanine substitutions are often used to avoid the possibility that mutants containing the more conservative serine substitution may retain some hydrolase activity. 
We report here that some active site cysteine to alanine substitutions can dramatically increase the affinity of DUBs for either free ubiquitin or polyubiquitin chains. This increase in affinity can confound the interpretation of in vivo experiments, since the mutant DUB is not only incapable of cleaving ubiquitin from substrates but has gained the ability to sequester free ubiquitin and polyubiquitin chains. Altering levels of free ubiquitin has been shown to give rise to off-target effects [17]. In addition, these mutant DUBs may stably associate with (poly)ubiquitinated substrates and thereby protect ubiquitin chains from cleavage by other DUBs or prevent interaction with ubiquitin receptors. The effects of such tight-binding DUB mutants thus have the potential to be highly diverse and unpredictable. In the case of the OTU DUB, A20, for example, the phenotype of an alanine substitution created a controversy over the physiological importance of its highly conserved DUB activity, since A20 containing alanine in place of cysteine was able to complement the wild-type version [18].

We show here that mutating the active site cysteine of human USP4 and yeast Ubp8 to alanine increases the affinity of the DUB for mono- or di-ubiquitin by $10-150$ fold. A similar effect of alanine substitution was previously found for the OTU enzyme, Cezanne (reference [19], supplementary data). The structure of the heterotetrameric SAGA DUB module containing Ubp8 ${ }^{\mathrm{C} 146 \mathrm{~A}}$ bound to free ubiquitin reveals the molecular basis for the increased affinity of monoubiquitin for the mutant enzyme. The alanine substitution alleviates steric hindrance by the active site cysteine sulfhydryl, allowing the C-terminal carboxylate of ubiquitin to form additional hydrogen bonds in the enzyme active site and thus accounts for the high affinity of the mutant enzyme for free ubiquitin. We show that substituting the active site cysteine of representative USP and OTU DUBs with arginine inactivates the enzymes while also disrupting binding to ubiquitin, generating an inert DUB domain. Based on these findings we strongly recommend that cell-based and in vivo studies of DUBs avoid the use of active site alanine substitutions, and to instead utilize substitutions such as arginine that ablate both enzymatic activity and ubiquitin binding. 


\section{RESULTS}

\section{Mutation of active site cysteine to alanine increases affinity of the SAGA DUB module for ubiquitin}

The yeast SAGA complex is a transcriptional coactivator that is involved in regulating mRNA transcription [20]. Among its activities is the removal of monoubiquitin from histone $\mathrm{H} 2 \mathrm{~B}$, which promotes transcription initiation and elongation [21]. The deubiquitinating activity of SAGA resides in a four-protein complex known as the DUB module, which comprises the USP family catalytic subunit, Ubp8, as well as Sgf11, Sus1 and the N-terminal $\sim 100$ residues of Sgf73 [22, 23]. Structural studies of the DUB module complexed with ubiquitin aldehyde and ubiquitinated nucleosomes have revealed the overall organization of the DUB module and how in interacts with substrate $[24,25]$. In addition to its ability to deubiquitinate histone $\mathrm{H} 2 \mathrm{~B}$, the DUB module can also cleave a variety of ubiquitin substrates in vitro including ubiquitin AMC and K48-linked diubiquitin [26]. The affinity of the DUB module for ubiquitinated nucleosome has been estimated at around $2 \mu \mathrm{M}$ [25] and the $K_{M}$ for the model substrate, ubiquitin-AMC, has been estimated at $24 \quad \mathrm{M}$ [26]; however, neither the $K_{M}$ nor binding affinity for other substrates is known.

In order to measure the affinity of the DUB module for other substrates using binding assays, we expressed and purified catalytically inactive versions of the DUB module containing Ubp8 with its active site cysteine, C146, substituted with either serine (C146S) or alanine (C146A). The absence of catalytic activity for both mutants was first verified in a ubiquitin-AMC cleavage assay (Fig. S1). We then measured the affinity of both mutant complexes for K48-linked diubiquitin using isothermal titration calorimetry (ITC) (Fig. 1E-F). Whereas DUB module containing Ubp8 ${ }^{\mathrm{C} 146 \mathrm{~S}}$ bound to $\mathrm{K} 48$ diubiquitin with a $\mathrm{K}_{d}$ of $4.6 \mu \mathrm{M}$, DUB module containing Ubp8 ${ }^{\mathrm{C} 146 \mathrm{~A}}$ bound to K48-linked diubiquitin with a $\mathrm{K}_{\mathrm{d}}$ of $0.47 \mu \mathrm{M}$, representing 10-fold tighter binding. We also measured the affinity of the reaction product, monoubiquitin, to DUB module containing either wild type or mutant Ubp8 (Fig. 1A-C). Whereas DUB module containing wild-type Ubp8 or Ubp8 ${ }^{\mathrm{C} 146 \mathrm{~S}}$ 
bound ubiquitin with a $\mathrm{K}_{d}$ of $13.9 \mathrm{M}$ and $12.8 \mathrm{M}$, respectively, the Ubp8 ${ }^{\mathrm{C} 146 \mathrm{~A}}$ mutant bound $\sim 30$-fold more tightly to monoubiquitin with a $\mathrm{Kd}$ of $0.43 \mathrm{M}$.

\section{Crystal structure of DUBm-Ubp $8^{\mathrm{C} 146 \mathrm{~A}}$ with Ub}

To determine the structural basis for the marked increase in affinity for free ubiquitin when the active site cysteine is substituted with alanine, we solved the crystal structure of the SAGA DUB module containing $U b p 8^{\mathrm{C} 146 \mathrm{~A}}$ bound to free ubiquitin at a resolution of $2.1 \AA$ (Table 1 and Fig. 2A). The overall fold and contacts with ubiquitin are virtually identical to those found in the structure of the wild type enzyme bound to ubiquitin aldehyde, aligning to an RMSD of $0.57 \AA$ [24]. The active site of the C146A mutant is virtually identical to that in the wild type apoenzyme [26], with no significant reordering of residues (Fig. 2B) [26]. In the Ubp8 ${ }^{\mathrm{C} 146 \mathrm{~A}}$ complex with free ubiquitin, the negatively charged carboxylate of the ubiquitin C-terminal Gly76 forms two hydrogen bonds with backbone amides from Ubp8 residues Thr145 and Ala146, as well as with active site residues, N146 and H427 (Fig. 2B and S2). Importantly, the observed position of the ubiquitin C-terminus would not be compatible with the presence of the wild-type active site residue, Cys146, since the sulfhydryl group would clash with the C-terminal residue of ubiquitin, Gly76 (Fig. 2C). The multiple hydrogen bonding interactions observed between the C-terminal carboxylate of ubiquitin and Ubp8 can therefore only occur when the active Cys146 is replaced with the smaller alanine side chain, thus explaining the higher affinity of DUB module-Ubp8 ${ }^{\mathrm{C} 146 \mathrm{~A}}$ for free ubiquitin as compared to the wild type enzyme.

\section{Active site cysteine to alanine substitution increases the affinity USP4 for ubiquitin}

Mutating the active site cysteine to alanine has an even more dramatic effect on the affinity of the human USP family DUB, USP4, for free ubiquitin. USP4 regulates a broad variety of cellular pathways, including TGF- $\beta$ and NF-KB signaling as well as splicing $[23,27,28]$. The affinity of USP4 for free ubiquitin was measured by fluorescence polarization using ubiquitin labeled with an $\mathrm{N}$-terminal fluorophore. As shown in Figure 
3, the $\mathrm{K}_{\mathrm{d}}$ of ubiquitin for the wild type enzyme is $92 \pm 21 \mathrm{nM}$ whereas USP4 containing an alanine substituted for the active site cysteine, C311, binds ubiquitin with $0.60 \pm 0.17$ nM affinity, a 150-fold difference [29]. The pre-steady-state kinetics of ubiquitin dissociation measured by fluorescence polarization in a stopped-flow device shows that the greater affinity of the USP $4^{\mathrm{C} 311 \mathrm{~A}}$ mutant is due to a dramatic decrease in off-rate (Fig. S3A). Interestingly, ubiquitin dissociation has been shown to be promoted by USP4 N-terminal DUSP-Ubl domain and to regulate USP4 activity [29]. The increase in affinity for the mutant enzyme is not unique to ubiquitin with a free $\mathrm{C}$-terminus, as ubiquitin conjugated to either an 18-mer peptide or C-terminal fluorophore also binds with similar affinity to USP4 ${ }^{\mathrm{C} 311 \mathrm{~A}}$ (Fig. S3C-D). Since the active sites of USP family DUBs are highly conserved, the increase in binding affinity is due a relief of steric clash, as is the case for Ubp8.

\section{Substitution of the catalytic cysteine with arginine disrupts ubiquitin binding in USP and OTU class DUBs}

We sought to identify alternative active site mutations that would abrogate catalytic activity as well as reduce the affinity of the inactive DUB polyubiquitin chains or ubiquitinated substrates. We reasoned that substituting the active site cysteine with arginine could both inactivate the enzyme and prevent ubiquitin binding because of the bulky nature of the side chain compared to cysteine. To test this hypothesis, we mutated the catalytic cysteine of Ubp8 to arginine and first verified that SAGA DUB module containing the mutant Ubp8 ${ }^{\mathrm{C} 146 \mathrm{R}}$ protein was inactive in a Ub-AMC cleavage assay (Fig. S1). The affinity of the DUB module containing Ubp8 ${ }^{\mathrm{C} 146 \mathrm{R}}$ for monoubiquitin as measured by ITC was comparable to that of the wild type protein (Fig. 1D).

Substitution of the active cysteine with arginine similarly reduces the affinity for polyubiquitin chains by the OTU family member, OTUD1, which is also a cysteine protease. This DUB preferentially cleaves K63-linked polyubiquitin chains [19], although the cellular function of this activity remains an area of active study. We measured the affinity of catalytic mutants of OTUD1 for fluorescently labelled K63-linked diubiquitin using a fluorescence polarization assay. While OTUD1 with an alanine substituted for 
the active site cysteine (OTUD1 ${ }^{\mathrm{C} 320 \mathrm{~A}}$ ) binds $\mathrm{K} 63$-linked diubiquitin with a $\mathrm{K}_{\mathrm{d}}$ of $\sim 40 \mu \mathrm{M}$, an arginine substitution, OTUD1 ${ }^{\mathrm{C} 320 \mathrm{R}}$, not only inactivated the enzyme but also completely abolished detectable binding to K63-linked diubiquitin (Fig. 4).

\section{OTUD1 active site arginine substitution overcomes artifacts of alanine substitution in cells}

As mentioned above, active site cysteine to alanine substitutions that markedly increase DUB affinity for mono or polyubiquitin may render these mutants less suitable for physiological studies. Since these cysteine to alanine mutants are essentially highaffinity ubiquitin binding proteins, these mutants could stabilize modified substrates and preferred chain types by protecting them from digestion by other DUBs or proteases. This problem should not arise if instead an arginine were substituted for the active site cysteine. To test this idea, we expressed HA-tagged wild-type OTUD1, OTUD1 ${ }^{\mathrm{C} 320 \mathrm{~A}}$ or OTUD $1^{\mathrm{C} 320 \mathrm{R}}$ in HEK293 cells, and analyzed K63-linked polyubiquitin in whole cell lysates by immunoblotting with an antibody specific for K63-polyubiquitin chains. As compared to cells expressing the wild-type protein, cells expressing OTUD $1^{\mathrm{C} 320 \mathrm{~A}}$ had increased levels of K63-linked polyubiquitin (Fig. 5). By contrast, cells expressing OTUD1 ${ }^{\mathrm{C} 320 \mathrm{R}}$ did not show enriched levels of K63-linked chains (Fig. 5). These results are consistent with the idea that the increase in polyubiquitin chains observed in the C320A mutant is due to the ability of this mutant to bind to polyubiquitin chains and protect them from cleavage by other DUBs. Our results also validate the utility of using a Cys to Arg substitution to generate a catalytically inactive DUB that will neither protect nor sequester polyubiquitin chains and ubiquitinated substrates.

\section{Discussion}

The surprisingly high affinity for ubiquitin exhibited by DUBs containing alanine substituted for the active site cysteine has important implications for cell-based assays in which catalytically inactive DUBs are expressed. We have found that cysteine to alanine substitutions in the USP DUBs, Ubp8 and USP4, dramatically increases their affinity for monoubiquitin (Figs. 1 and 3). Similarly, cysteine to alanine substitutions in 
the active sites of Ubp8 and the OTU class DUB, OTUD1, also increase DUB affinity for polyubiquitin (Figs. 1 and 4). The equilibrium dissociation constants of these mutant DUBs are significantly lower than cellular concentrations of their substrates, with an estimated concentration of free ubiquitin in the cell of $4-50 \mu \mathrm{M}$ [30] and a concentration of polyubiquitin at a fraction of that [31]. The mutant DUBs are therefore expected to bind avidly to free ubiquitin and polyubiquitin chains when expressed in cells. Particularly in experiments where the mutant DUBs are overexpressed, there is the risk that cellular consequences ascribed to a lack of catalytic activity in a particular DUB may instead be due to the ability of the mutant DUB to protect polyubiquitin chains from cleavage or to a difference in free ubiquitin available to ubiquitin conjugating enzymes.

We have presented an alternative to alanine substitutions that is equally effective in abrogating DUB activity while having the advantage of preventing ubiquitin binding. In binding assays of Ubp8 and OTUD1, we show that replacing the active site cysteine with arginine inactivates ubiquitin hydrolase activity while also rendering the enzyme incapable of binding ubiquitin detectably (Figs. 1D and 4). We speculate that the ability of arginine substitutions to abolish ubiquitin binding to OTU and USP catalytic domains may be explained by the ability of the arginine side chain to partially occupy the binding site for the C-terminal ubiquitin Gly-Gly in these DUBs. Previous data has indicated that the correct orientation of the ubiquitin C-terminal tail in the DUB S1 site is essential for efficient cleavage $[32,33]$. An arginine side chain may mimic these interaction in cis, to prevent these important substrate interactions.

While we did not test other active site substitutions, it is expected that other amino acids with side chains that are significantly bulkier than cysteine would similarly block ubiquitin binding in addition to inactivating the enzyme. However, care should be taken to avoid hydrophobic side chains that could cause the protein to aggregate, or side chains that are bulky or beta-branched that could interfere with proper protein folding due to steric clashes with the neighboring protein backbone. Since lysine can be ubiquitinated and is also subject to many other post-translational modifications, this substitution should also be avoided. 
We recommend that all cell-based and in vivo studies of cysteine protease DUBs avoid alanine substitutions of the active site cysteine and instead utilize arginine substitutions to study effects of inactivating the enzyme. Although the arginine substitution was only tested here on DUBs from the USP and OTU class, it is possible that an arginine would similarly interfere with ubiquitin binding to members of the UCH, MJD, and MINDY cysteine protease families, in addition to inactivating the enzyme. Adopting this practice can mitigate any spurious, off-target effects and ensure that any observed phenotypes or changes are due to loss of DUB activity alone rather than an increase in affinity for ubiquitin. 


\section{Acknowledgements}

We would like to thank Tycho E.T. Mevissen for help with OTU biophysics.

Supported by grants GM095822 and GM109102 from the National Institute of General Medical Sciences (C.W.). The work in the T.K.S. lab has been supported by the European Research Council (249997). The D.K. lab is supported by the Medical Research Council [U105192732], the European Research Council [309756, 724804], the Michael J. Fox Foundation and the Lister Institute for Preventive Medicine. M.C. and M.S. were supported by EMBO Long Term Fellowships. Use of the Stanford Synchrotron Radiation Lightsource, SLAC National Accelerator Laboratory, is supported by the U.S. Department of Energy, Office of Science, Office of Basic Energy Sciences under Contract No. DE-AC02-76SF00515. The SSRL Structural Molecular Biology Program is supported by the DOE Office of Biological and Environmental Research, and by the National Institutes of Health, National Institute of General Medical Sciences (including P41GM103393). 


\section{Materials and Methods}

\section{Cloning, protein expression and purification}

Rosetta 2(DE3) pLysS cells (EMD Millipore, Merck KGaA, Darmstadt, Germany) were transformed with three plasmids encoding (1) Ubp8 ${ }^{\mathrm{WT}}$, Ubp8 ${ }^{\mathrm{C} 146 \mathrm{~A}}, \mathrm{Ubp}^{\mathrm{C} 146 \mathrm{~S}}$ or Ubp8 $^{\mathrm{C} 146 \mathrm{R}}$ (pET-32a, EMD Millipore), (2) Sus1 (pRSF-1, EMD Millipore), and (3) Sgf73 ${ }^{(1-}$ 96), (pCDFDuet-1 MCSII, EMD Millipore), which was cloned into the same vector as Sgf11 (pCDFDuet-1 MCSI, EMD Millipore). All versions of the DUBm complex were coexpressed and purified using the previously reported protocol for the expression and purification of wild-type DUBm [24]. Untagged ubiquitin (pET3a) was expressed in Rosetta 2 cells and after lysis, was treated with $1 \% \mathrm{v} / \mathrm{v}$ perchloric acid to precipitate cellular proteins. The supernatant, containing ubiquitin, was dialyzed overnight into 50 $\mathrm{mM}$ sodium acetate $\mathrm{pH} 4.5$, then run on a HiTrap SP column and eluted over a $0-600$ $\mathrm{mM} \mathrm{NaCl}$ gradient in $50 \mathrm{mM}$ sodium acetate $\mathrm{pH}$ 4.5. Pure fractions were pooled and buffer exchanged by gel filtration on a HiLoad S75 column into 20 mM HEPES pH 7.5, $50 \mathrm{mM} \mathrm{NaCl}, 1 \mathrm{mM}$ DTT.

USP4(8-925) wild-type and C311A mutant were expressed and purified as in [29]. pOPINK-OTUD1 catalytic domain (residues 287-435) was transformed into E.coli Rosetta 2 pLysS (Novagen) and grown to OD 0.6 followed by induction with $0.2 \mathrm{mM}$ IPTG overnight at $20^{\circ} \mathrm{C}$. Cells were lysed by sonication in buffer A (50 mM Tris, $50 \mathrm{mM}$ $\mathrm{NaCl}, 5 \mathrm{mM}$ DTT, $\mathrm{pH} 8.5$ ), the lysate was cleared by centrifugation (44000 x $\mathrm{g}$ for 30 $\min , 4^{\circ} \mathrm{C}$ ) and subjected to a glutathione resin (GE Healthcare). The resin was washed with cold buffer B buffer (50 mM Tris, $500 \mathrm{mM} \mathrm{NaCl,} 5 \mathrm{mM}$ DTT, pH 8.5) and subsequently with cold buffer A. The GST-tag was removed by overnight incubation at $4^{\circ} \mathrm{C}$ with GST-tagged 3C Precision protease in buffer A. Eluted protein was further purified by anion-exchange chromatography and gel filtration in buffer $A$.

\section{Fluorescence Polarization Assays}


All USP4 pre-steady-state and equilibrium fluorescence polarization assays were performed as described in [29]. Ubiquitin conjugated to lysine-glycine and to a SMAD4derived peptide [29] were a gift of Huib Ovaa.

Binding assays for OTUD1 interactions with Lys63-linked chains were performed using Lys63-linked diUb that was fluorescently labeled by a FIAsH-tag on the proximal ubiquitin (21). For this, diUb chains were diluted to $80 \mathrm{nM}$ in FIAsH buffer (50 mM Tris, $50 \mathrm{mM} \mathrm{NaCl}, 0.1 \%$ $\beta$-mercaptoethanol, $\mathrm{pH} 7.6)$, and OTUD1 ${ }^{\mathrm{C} 320 \mathrm{~A}}$ and OTUD1 ${ }^{\mathrm{C} 320 \mathrm{R}}$ were serially diluted in FIAsH buffer to the indicated concentration range. $10 \mu$ of fluorescent diUb was mixed with equal volume of OTUD $1^{\mathrm{C} 320 \mathrm{~A}}$ and OTUD1 ${ }^{\mathrm{C} 320 \mathrm{R}}$ at different concentrations and incubated in room temperature for $1 \mathrm{~h}$ before measurement. Fluorescence polarization was measured in 384 well format employing a Pherastar FS plate reader, using a fluorescence polarization module with excitation and emission wavelengths at $485 \mathrm{~nm}$ and $520 \mathrm{~nm}$ respectively. A control was used for either linear dior triUb molecules where $10 \mu \mathrm{l}$ of FIAsH buffer was added instead. This control was also used for the normalization of anisotropy reading. All binding assays were performed in triplicate.

\section{Ubiquitin-AMC hydrolysis Assay}

Assays were conducted in 384-well black polystyrene micro-plates at $30^{\circ} \mathrm{C}$ in a POLARstar Omega plate reader (BMG Labtech, Cary, NC) using an excitation wavelength of $385 \mathrm{~nm}$ and emission wavelength of $460 \mathrm{~nm}$. Reactions were performed in DUBm assay buffer containing 50 mM HEPES, pH 7.6, $150 \mathrm{mM} \mathrm{NaCl}, 5 \mu \mathrm{M} \mathrm{ZnCl}_{2}, 5$ $\mathrm{mM}$ dithiothreitol (DTT) and 7.5\% DMSO. The wild type DUBm and Ubp8 mutants were held at a concentration of $125 \mathrm{nM}$. Ubiquitin-AMC (Boston Biochem, Cambridge, MA) was diluted into assay buffer and incubated at $30^{\circ} \mathrm{C}$ for $10 \mathrm{~min}$ inside the plate reader. 3 $\mu \mathrm{l}$ of recombinant DUBm was also pre-incubated at $30^{\circ} \mathrm{C}$ for $10 \mathrm{~min}$ before mixing with diluted ubiquitin-AMC buffer to a total volume of $30 \mu$. The release of AMC was followed at $460 \mathrm{~nm}$, and the first $0-60 \mathrm{~s}$ of data were used to fit initial rate. 


\section{Isothermal Titration Calorimetry (ITC)}

ITC measurements were performed by using a Microcal (Amherst, MA) ITC200 calorimeter at $25^{\circ} \mathrm{C}$. DUBm wild type, Ubp8 mutant complexes, K48-linked diubiquitin and ubiquitin samples were buffered with $20 \mathrm{mM} \mathrm{HEPES,} \mathrm{pH} \mathrm{7,} 150 \mathrm{mM} \mathrm{NaCl}, 5 \mathrm{nM}$ $\mathrm{ZnCl}_{2}, 0.5 \mathrm{mM}$ Tris (2-Carboxyethyl) phosphine hydrochloride (TCEP) and thoroughly degassed before use. The protein concentrations were determined by amino acid analysis. The sample cell $(0.22 \mathrm{ml})$ contained either $30 \mu \mathrm{M}$ DUBm-Ubp8 ${ }^{\mathrm{WT}}$ or DUBm Ubp8 mutant. A total of 20 injections of $40 \mu \mathrm{l}$ of $0.3 \mathrm{mM} \mathrm{K} 48$-linked diubiquitin or monoubiquitin were carried out at $180 \mathrm{~s}$ intervals. The heat generated due to dilution of the titrants was subtracted for baseline correction. The baseline-corrected data were analyzed with Microcal Origin Ver. 7.0 software. All experiments were duplicated.

\section{Protein crystallization}

Protein crystals were grown from a complex of $7 \mathrm{mg} / \mathrm{mL}$ DUBm-Ubp8 ${ }^{\mathrm{C} 146 \mathrm{~A}}$ and 1.8 $\mathrm{mg} / \mathrm{mL}$ ubiquitin that was incubated on ice for 30 minutes prior to screening. Complex crystals were grown by hanging drop vapor diffusion using a 1:1 ratio of protein to mother liquor. Crystals grew within 2 days at $20^{\circ} \mathrm{C}$ in $17 \%$ PEG3350, $0.1 \mathrm{M} \mathrm{HEPES} \mathrm{pH}$ 7.0, $0.1 \mathrm{M}$ ammonium sulfate. Crystals were looped and cryoprotected by stepwise incubation in mother liquor containing increasing concentrations of PEG3350 (17-33\%), then flash frozen in liquid nitrogen.

\section{Data collection, structure determination and refinement}

X-ray diffraction data for the DUBm-Ubp8 ${ }^{\mathrm{C} 146 \mathrm{~A}}$ and ubiquitin complex were collected at Stanford Synchrotron Radiation Lightsource beamline BL12-2. Data were collected on a Pilatus detector using a $10 \mu \mathrm{m}$ beam at $50 \%$ transmission taking 1 second exposures with $0.25^{\circ}$ oscillations over $180^{\circ}$. During data collection, the crystal rotated out of the beam, therefore frames 200-300 out of 720 total frames were discarded during data reduction and scaling. Data reduction, scaling, and merging were done in XDS and 
Aimless [34]). A 2.1 Å structure was determined by molecular replacement in Phaser (Phenix) using the coordinates of the wild-type DUBm bound to ubiquitin aldehyde (PDB ID: 3MHS) as the search model[24, 35]. The structure was refined in PHENIX and Coot was used for manual model building[35, 36]. Data collection and refinement statistics are shown in Table 1. PyMOL Version 1.5.0.4 (Schrödinger, LLC) was used to generate all structure figures. Coordinates have been deposited in the Protein Data Bank with ID 6AQR.

\section{Immunoblotting and immunoprecipitation}

Full-length, N-terminally HA-tagged OTUD1 constructs (WT, C320A, C320R) were cloned into pcDNA3.1 vector and transiently expressed in HEK293 cells. Two days after transfection cells were washed twice in cold PBS and scraped on ice in lysis buffer (50 mM Tris- $\mathrm{HCl}$ pH 7.5, 150 mM NaCl, 1\% NP-40) containing protease inhibitor cocktail (Roche) and $20 \mathrm{mM} \mathrm{N}$-ethylmaleimide, and incubated for 30 min on ice. Samples were subsequently cleared by centrifugation for $10 \mathrm{~min}$ at $16,000 \times \mathrm{g}$ at $4^{\circ} \mathrm{C}$. For immunoblotting, equivalent amounts of cell lysates were separated on $4-12 \%$ BisTris gel, transferred to nitrocellulose membranes, and incubated with the indicated antibodies. The signal was visualized with Pierce ECL Western Blotting Substrate (Thermo Scientific) and exposed on X-ray film.

\section{Antibodies}

The following antibodies were used in immunoblotting or immunoprecipitation: rat monoclonal anti-HA (clone 3F10, Sigma-Aldrich), rabbit monoclonal anti-Ub K63 (clone Apu3, Millipore) and mouse anti-GAPDH (clone D4C6R, Cell Signaling). 


\begin{tabular}{|c|c|}
\hline Wavelength $(\AA)$ & 0.979 \\
\hline Resolution ( $\AA$ ) & 2.10 \\
\hline Unique reflections & 54195 \\
\hline Redundancy & $5.7(5.7)$ \\
\hline Completeness (\%) & $99.2(99.7)$ \\
\hline Average $\mathrm{I} / \sigma(\mathrm{I})$ & $13.3(3.0)$ \\
\hline$R_{\text {merge }}$ & $0.093(0.572)$ \\
\hline$R_{\text {meas }}$ & $0.103(0.630)$ \\
\hline $\mathrm{R}_{\text {pim }}$ & $0.043(0.259)$ \\
\hline $\mathrm{CC} 1 / 2$ & $0.998(0.857)$ \\
\hline $\mathrm{CC}^{*}$ & $0.999(0.961)$ \\
\hline \multicolumn{2}{|l|}{ Refinement Statistics } \\
\hline $\begin{array}{l}\text { Space Group and } \\
\text { Unit Cell }(\AA)\end{array}$ & $\begin{array}{c}P 2{ }_{1} 2{ }_{2}{ }_{1} \\
a=78.8, b=103.2, c=112.8\end{array}$ \\
\hline Molecules per asymmetric unit & 1 \\
\hline $\mathrm{R}_{\text {work }}(\%)$ & 20.1 \\
\hline $\mathrm{R}_{\text {free }}(\%)$ & 24.9 \\
\hline Rmsd bonds ( $\AA$ ) & 0.0198 \\
\hline Rmsd angles $\left({ }^{\circ}\right)$ & 1.855 \\
\hline Protein atoms & 6302 \\
\hline Zinc ions & 8 \\
\hline Average $B\left(\AA^{2}\right)$ & 40.3 \\
\hline
\end{tabular}




\section{References}

1. Clague, M.J., J.M. Coulson, and S. Urbe, Cellular functions of the DUBs. J Cell Sci, 2012. 125(Pt 2): p. 277-86.

2. Takeuchi, T., et al., Link between the ubiquitin conjugation system and the ISG15 conjugation system: ISG15 conjugation to the UbcH6 ubiquitin E2 enzyme. J Biochem, 2005. 138(6): p. 711-9.

3. Reyes-Turcu, F.E., K.H. Ventii, and K.D. Wilkinson, Regulation and cellular roles of ubiquitin-specific deubiquitinating enzymes. Annu Rev Biochem, 2009. 78: p. 363-97.

4. Clague, M.J., et al., Deubiquitylases from genes to organism. Physiol Rev, 2013. 93(3): p. 1289-315.

5. Abdul Rehman, S.A., et al., MINDY-1 Is a Member of an Evolutionarily Conserved and Structurally Distinct New Family of Deubiquitinating Enzymes. Mol Cell, 2016. 63(1): p. 146-55.

6. Pfoh, R., I.K. Lacdao, and V. Saridakis, Deubiquitinases and the new therapeutic opportunities offered to cancer. Endocr Relat Cancer, 2015. 22(1): p. T35-54.

7. Heideker, J. and I.E. Wertz, DUBs, the regulation of cell identity and disease. Biochem J, 2015. 465(1): p. 1-26.

8. Singhal, S., M.C. Taylor, and R.T. Baker, Deubiquitylating enzymes and disease. BMC Biochem, 2008. 9 Suppl 1: p. S3.

9. Fraile, J.M., et al., Deubiquitinases in cancer: new functions and therapeutic options. Oncogene, 2012. 31(19): p. 2373-88.

10. Harrigan, J.A., et al., Deubiquitylating enzymes and drug discovery: emerging opportunities. Nat Rev Drug Discov, 2017.

11. Sippl, W., V. Collura, and F. Colland, Ubiquitin-specific proteases as cancer drug targets. Future Oncol, 2011. 7(5): p. 619-32.

12. Komander, D., M.J. Clague, and S. Urbe, Breaking the chains: structure and function of the deubiquitinases. Nat Rev Mol Cell Biol, 2009. 10(8): p. 550-63.

13. Nakada, S., et al., Non-canonical inhibition of DNA damage-dependent ubiquitination by OTUB1. Nature, 2010. 466(7309): p. 941-6.

14. Juang, Y.C., et al., OTUB1 co-opts Lys48-linked ubiquitin recognition to suppress E2 enzyme function. Mol Cell, 2012. 45(3): p. 384-97.

15. Wiener, R., et al., The mechanism of OTUB1-mediated inhibition of ubiquitination. Nature, 2012. 483(7391): p. 618-22.

16. Zhao, Y., et al., Noncanonical regulation of alkylation damage resistance by the OTUD4 deubiquitinase. EMBO J, 2015. 34(12): p. 1687-703. 
17. Dantuma, N.P., et al., A dynamic ubiquitin equilibrium couples proteasomal activity to chromatin remodeling. J Cell Biol, 2006. 173(1): p. 19-26.

18. De, A., et al., The deubiquitinase activity of $A 20$ is dispensable for NF-kappaB signaling. EMBO Rep, 2014. 15(7): p. 775-83.

19. Mevissen, T.E.T., et al., Molecular basis of Lys11-polyubiquitin specificity in the deubiquitinase Cezanne. Nature, 2016. 538(7625): p. 402-405.

20. Rodriguez-Navarro, S., Insights into SAGA function during gene expression. EMBO Rep, 2009. 10(8): p. 843-50.

21. Henry, K.W., et al., Transcriptional activation via sequential histone $H 2 B$ ubiquitylation and deubiquitylation, mediated by SAGA-associated Ubp8. Genes Dev, 2003. 17(21): p. 2648-63.

22. Kohler, A., et al., Yeast Ataxin-7 links histone deubiquitination with gene gating and mRNA export. Nat Cell Biol, 2008. 10(6): p. 707-15.

23. Lee, K.K., et al., Yeast Sgf73/Ataxin-7 serves to anchor the deubiquitination module into both SAGA and Slik(SALSA) HAT complexes. Epigenetics Chromatin, 2009. 2(1): p. 2.

24. Samara, N.L., et al., Structural insights into the assembly and function of the SAGA deubiquitinating module. Science, 2010. 328(5981): p. 1025-9.

25. Morgan, M.T., et al., Structural basis for histone H2B deubiquitination by the SAGA DUB module. Science, 2016. 351(6274): p. 725-8.

26. Samara, N.L., A.E. Ringel, and C. Wolberger, A role for intersubunit interactions in maintaining SAGA deubiquitinating module structure and activity. Structure, 2012. 20(8): p. 1414-24.

27. Song, E.J., et al., The Prp19 complex and the Usp4Sart3 deubiquitinating enzyme control reversible ubiquitination at the spliceosome. Genes Dev, 2010. 24(13): p. 1434-47.

28. Zhang, L., et al., USP4 is regulated by AKT phosphorylation and directly deubiquitylates TGF-beta type I receptor. Nat Cell Biol, 2012. 14(7): p. 717-26.

29. Clerici, M., et al., The DUSP-Ubl domain of USP4 enhances its catalytic efficiency by promoting ubiquitin exchange. Nat Commun, 2014. 5: p. 5399.

30. Clague, M.J., C. Heride, and S. Urbe, The demographics of the ubiquitin system. Trends Cell Biol, 2015. 25(7): p. 417-26.

31. Kaiser, S.E., et al., Protein standard absolute quantification (PSAQ) method for the measurement of cellular ubiquitin pools. Nat Methods, 2011. 8(8): p. 691-6.

32. Bekes, M., et al., DUB-resistant ubiquitin to survey ubiquitination switches in mammalian cells. Cell Rep, 2013. 5(3): p. 826-38.

33. Drag, M., et al., Positional-scanning fluorigenic substrate libraries reveal unexpected specificity determinants of DUBs (deubiquitinating enzymes). Biochem J, 2008. 415(3): p. 367-75. 
34. Winn, M.D., et al., Overview of the CCP4 suite and current developments. Acta Crystallogr D Biol Crystallogr, 2011. 67(Pt 4): p. 235-42.

35. Adams, P.D., et al., PHENIX: a comprehensive Python-based system for macromolecular structure solution. Acta Crystallogr D Biol Crystallogr, 2010. 66(Pt 2): p. 213-21.

36. Emsley, P. and K. Cowtan, Coot: model-building tools for molecular graphics. Acta Crystallogr D Biol Crystallogr, 2004. 60(Pt 12 Pt 1): p. 2126-32. 


\section{Figures}

\section{Figure 1}

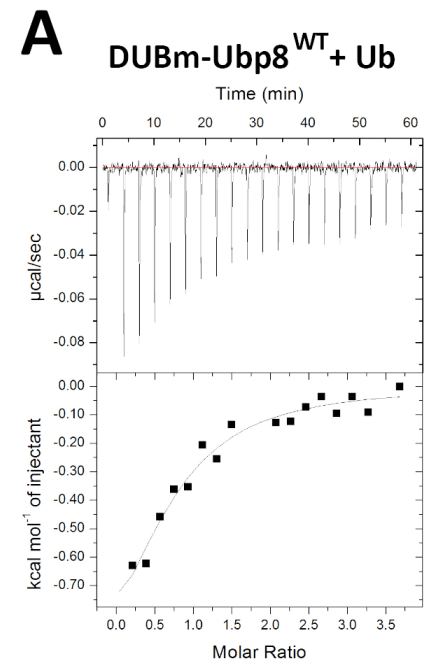

D DUBm-Ubp8 ${ }^{\mathrm{C146R}}+\mathrm{Ub}$

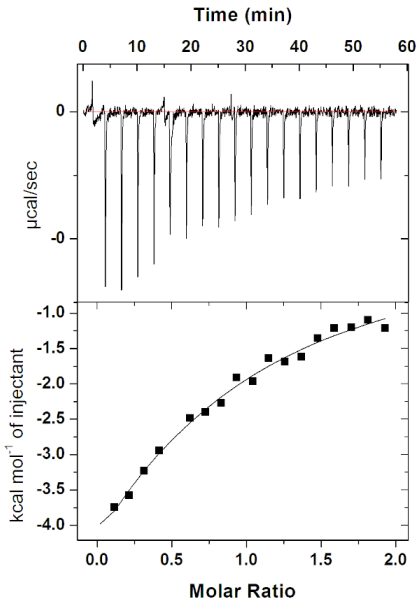

B

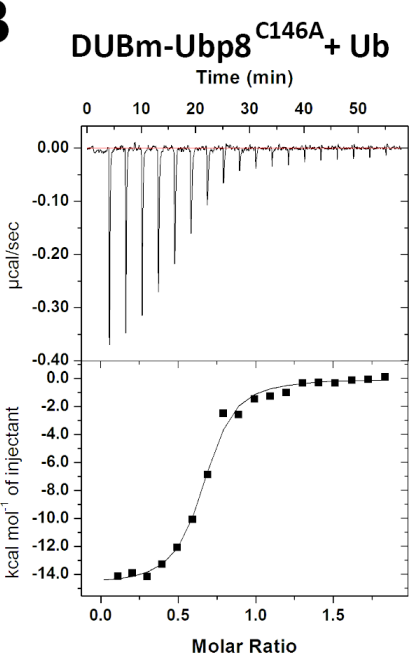

$\mathbf{E}$

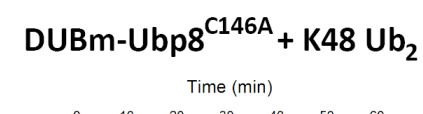

C DUBm-Ubp8 ${ }^{\mathrm{C} 146 \mathrm{~S}}+\mathrm{Ub}$

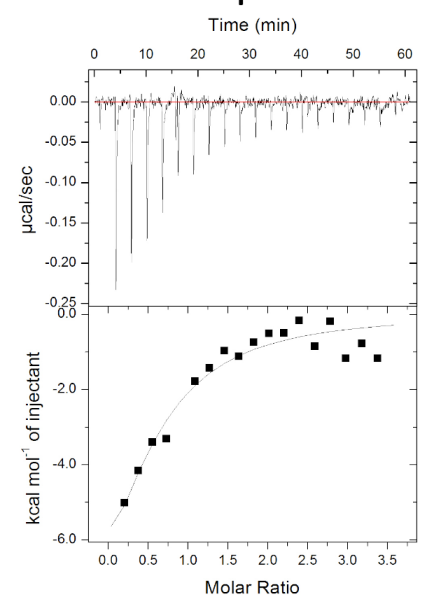

$F_{\text {DUBm-Ubp8 }}^{\mathrm{C}^{1465}+\mathrm{K} 48 \mathrm{Ub}_{2}}$

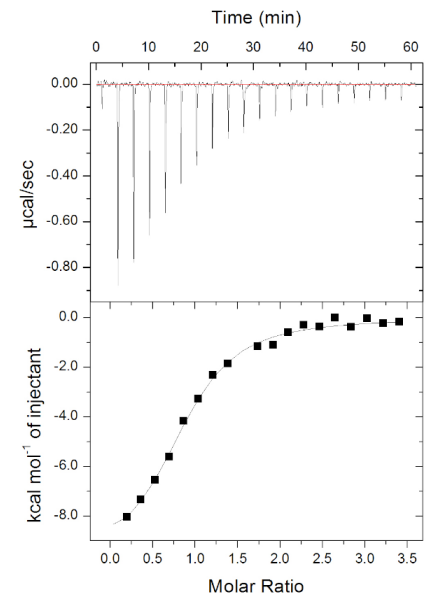

Figure 1. Isothermal titration calorimetry assays of SAGA DUB module binding to K48 diubiquitin or monoubiquitin. (A) Binding of wild type DUBm-Ubp8 to monoubiquitin. (B) Binding of DUBmUbp8 ${ }^{\mathrm{C} 146 \mathrm{~A}}$ to monoubiquitin. (C) Binding of DUBmUbp8 ${ }^{\mathrm{C} 146 \mathrm{~S}}$ to monoubiquitin. (D) Binding of DUBm-Ubp8 ${ }^{\mathrm{C} 146 \mathrm{R}}$ to monoubiquitin. (E) Binding of DUBm-Ubp8 ${ }^{\mathrm{C} 146 \mathrm{~A}}$ to $\mathrm{K} 48$ diubiquitin. $(\mathrm{F})$ Binding of DUBm-Ubp8 ${ }^{\mathrm{C} 146 \mathrm{~S}}$ to $\mathrm{K} 48$ diubiquitin. 


\section{Figure 2}
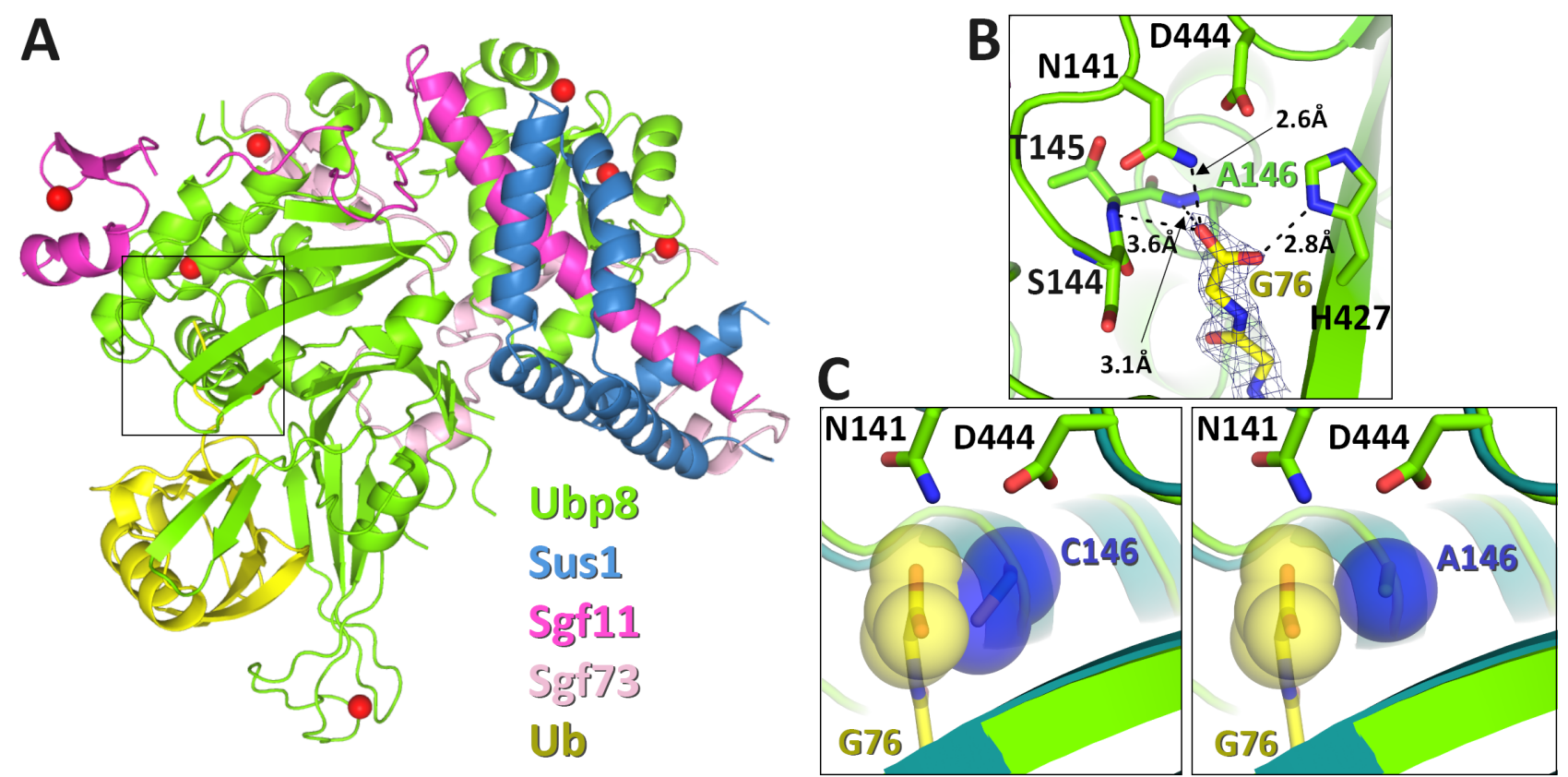

Figure 2. X-ray crystal structure of SAGA DUB module mutant DUBm-Ubp8 ${ }^{\mathrm{C} 146 \mathrm{~A}}$ bound to monoubiquitin. (A) Overall structure of complex showing Ubp8 (green) with ubiquitin (yellow) bound to the USP domain. (B) Hydrogen bonding contacts between the C-terminal carboxylate of ubiquitin and Ubp8. (C) In grey spheres, van der Waals radii of C146 (teal) and A146 (green) in steric proximity of ubiquitin's C-terminal carboxylate (yellow). DUBm-Ubp8 ${ }^{\mathrm{WT}}$ structure is shown in teal (PDB ID $3 \mathrm{MHH}$ ) and DUBm-Ubp8 ${ }^{\mathrm{C} 146 \mathrm{~A}}$ is shown in green. 


\section{Figure 3}

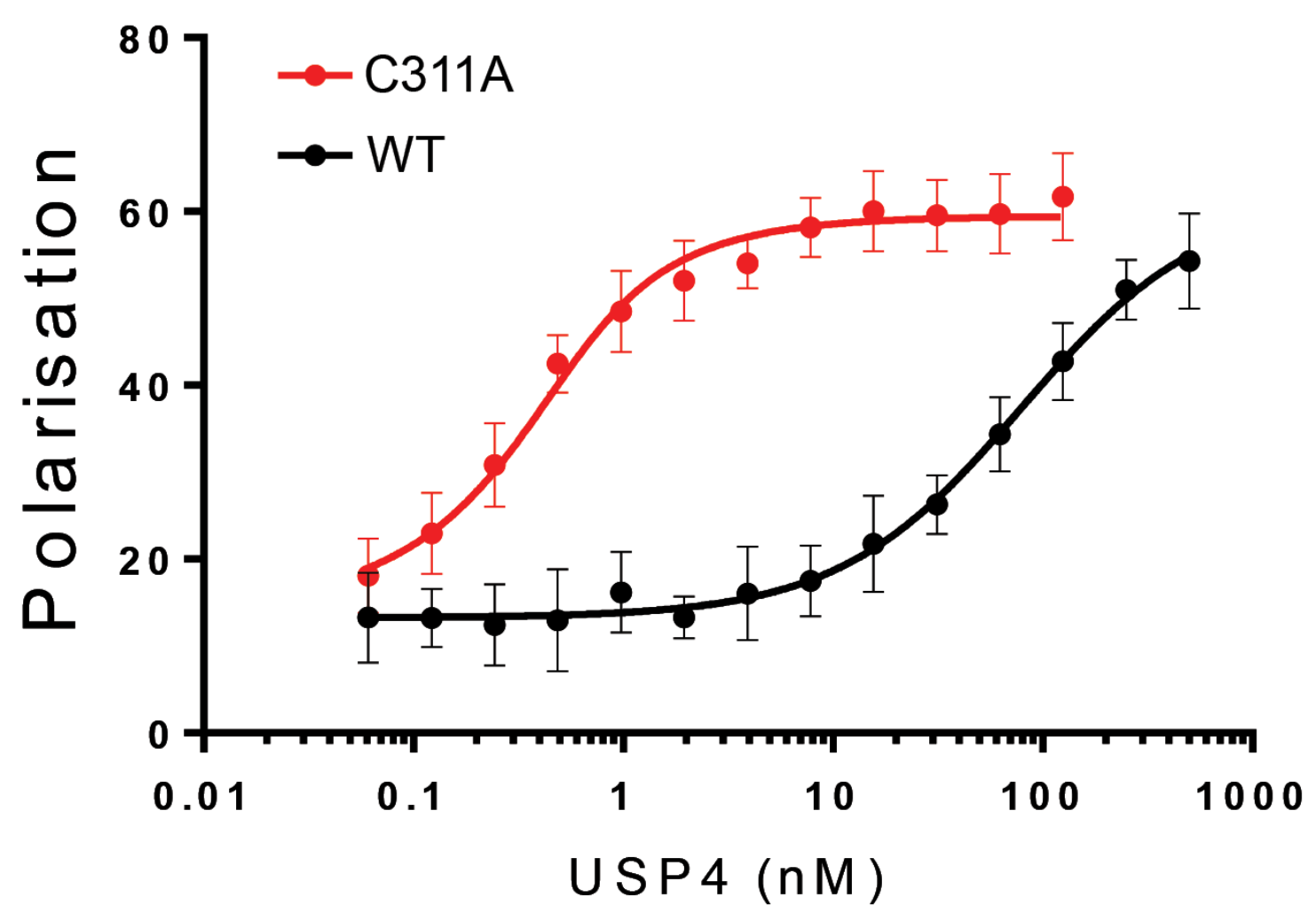

Figure 3. Equilibrium binding of USP4 WT and C311A to TAMRA-labeled monoubiquitin. Binding was measured by fluorescence polarization using $\mathrm{N}$-terminally TAMRA-labeled monoubiquitin. The dissociation constants for ubiquitin binding to USP4 WT and C311A are $92 \pm 21 \mathrm{nM}$ (Clerici et. al, 2014) and $0.60 \pm 0.17 \mathrm{nM}$, respectively. 


\section{Figure 4}

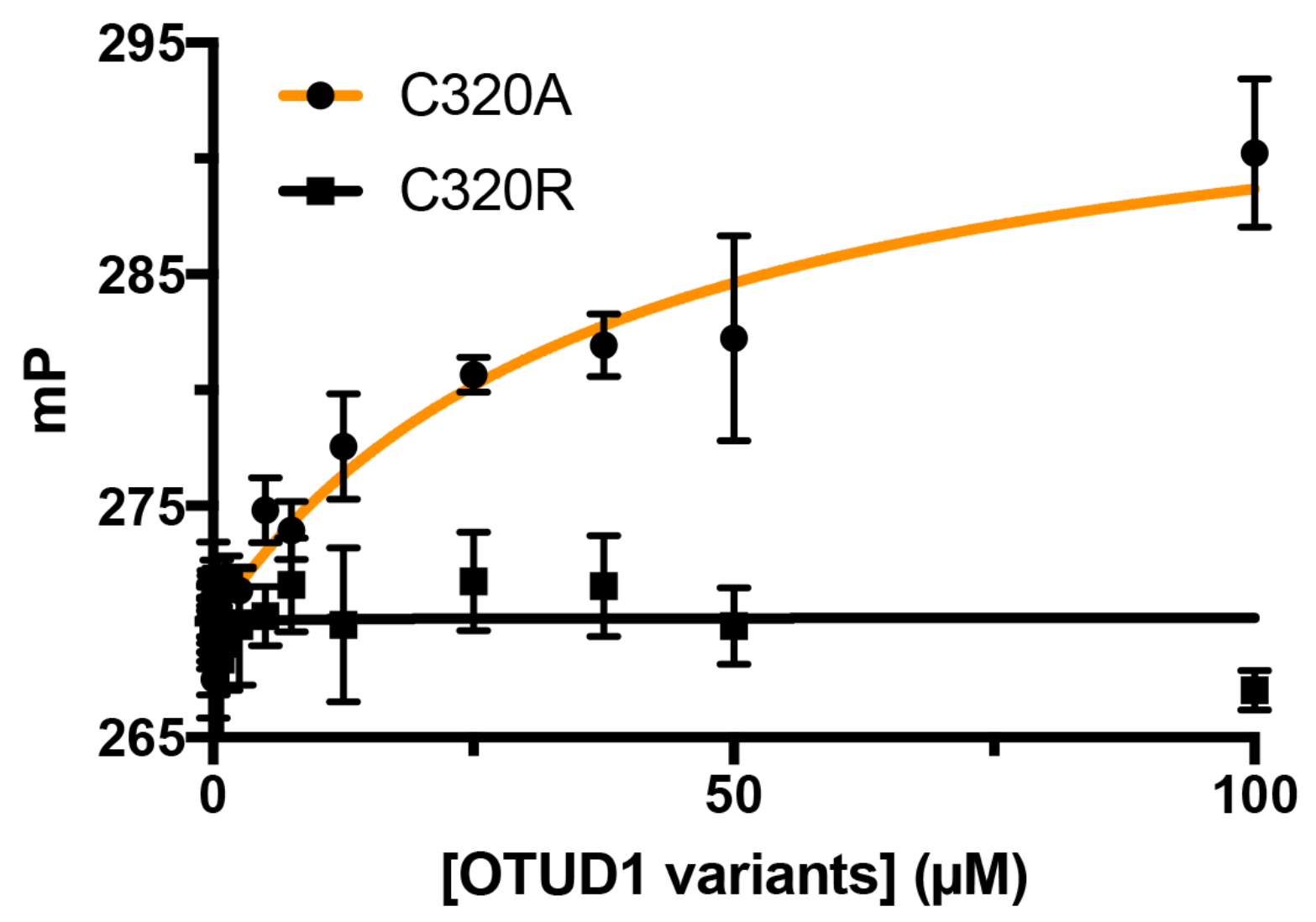

Figure 4: Equilibrium binding of OTUD1 C320A and C320R to K63-linked diubiquitin. Binding was measured by fluorescence polarization using $\mathrm{FIAsH}$ tagged K63-linked diubiquitin in which the proximal ubiquitin was fluorescently-labeled. 


\section{Figure 5}
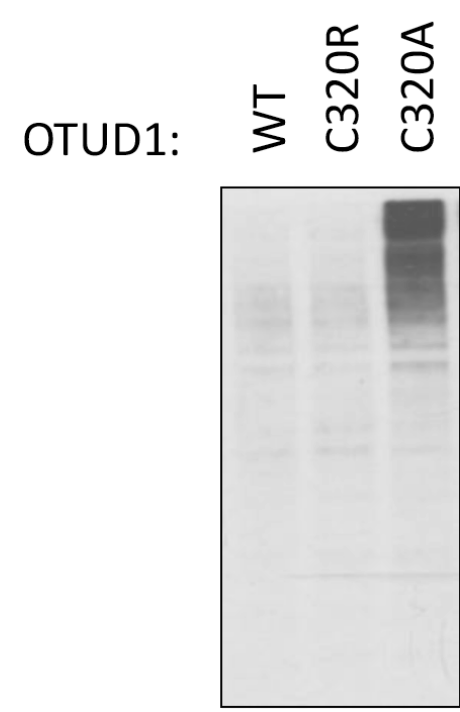

Ub K63

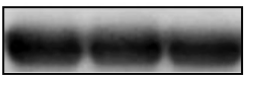

HA-OTUD1

GAPDH

Figure 5: Stabilization of ubiquitin chains by OTUD1 C320A in vivo. Whole cell lysates of HEK293 cells expressing HA-tagged OTUD1 WT, C320R, and C320A were immunoblotted with indicated antibodies. 


\section{Supplementary Figures}

Figure $\mathbf{S 1}$

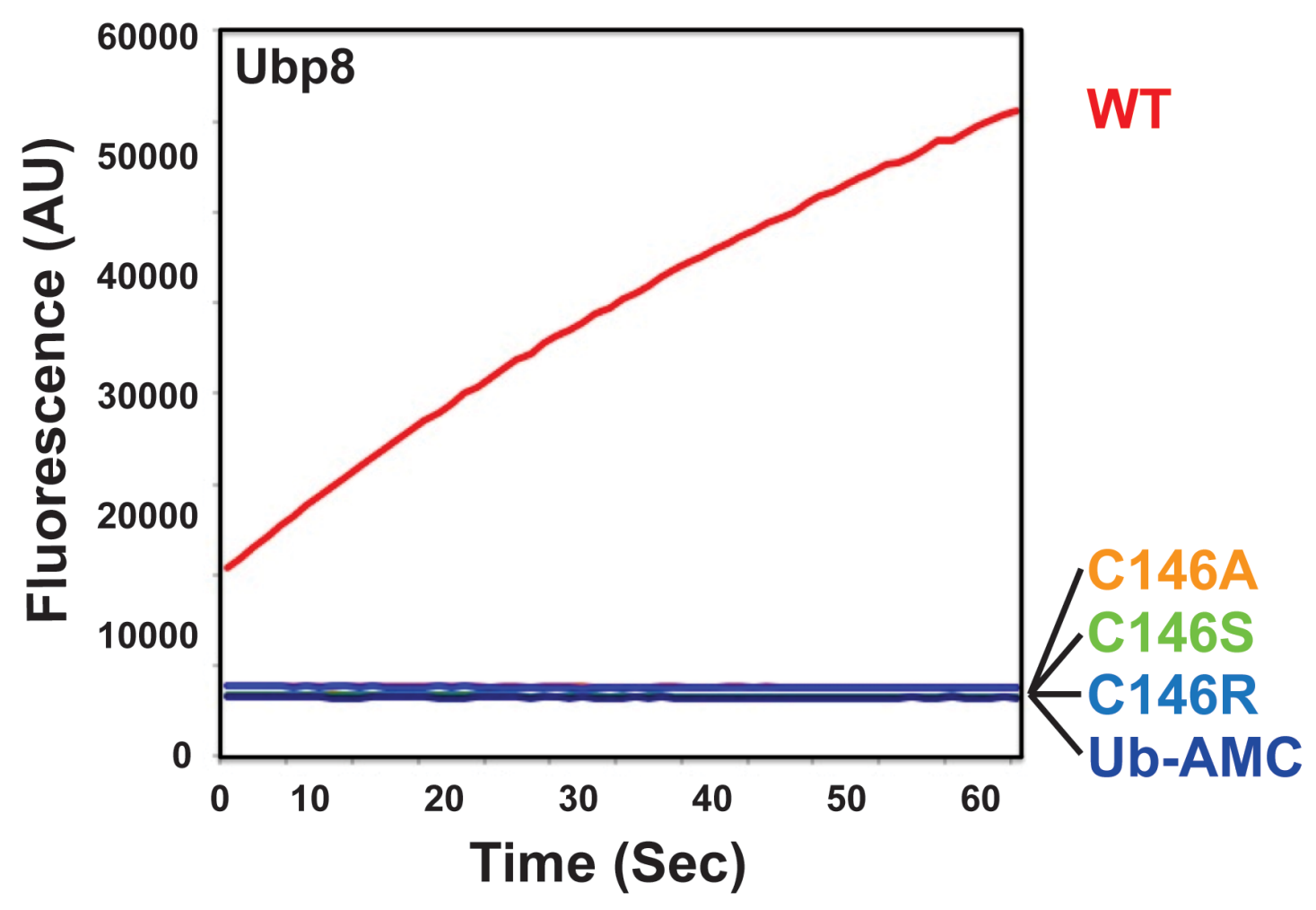

Figure S1: Catalytic activity of DUBm-Ubp8 mutants. Progress curve of Ub-AMC cleavage by $125 \mathrm{nM}$ DUBm-Ubp8 ${ }^{\mathrm{WT}}$ or mutants. 


\section{Figure S2}

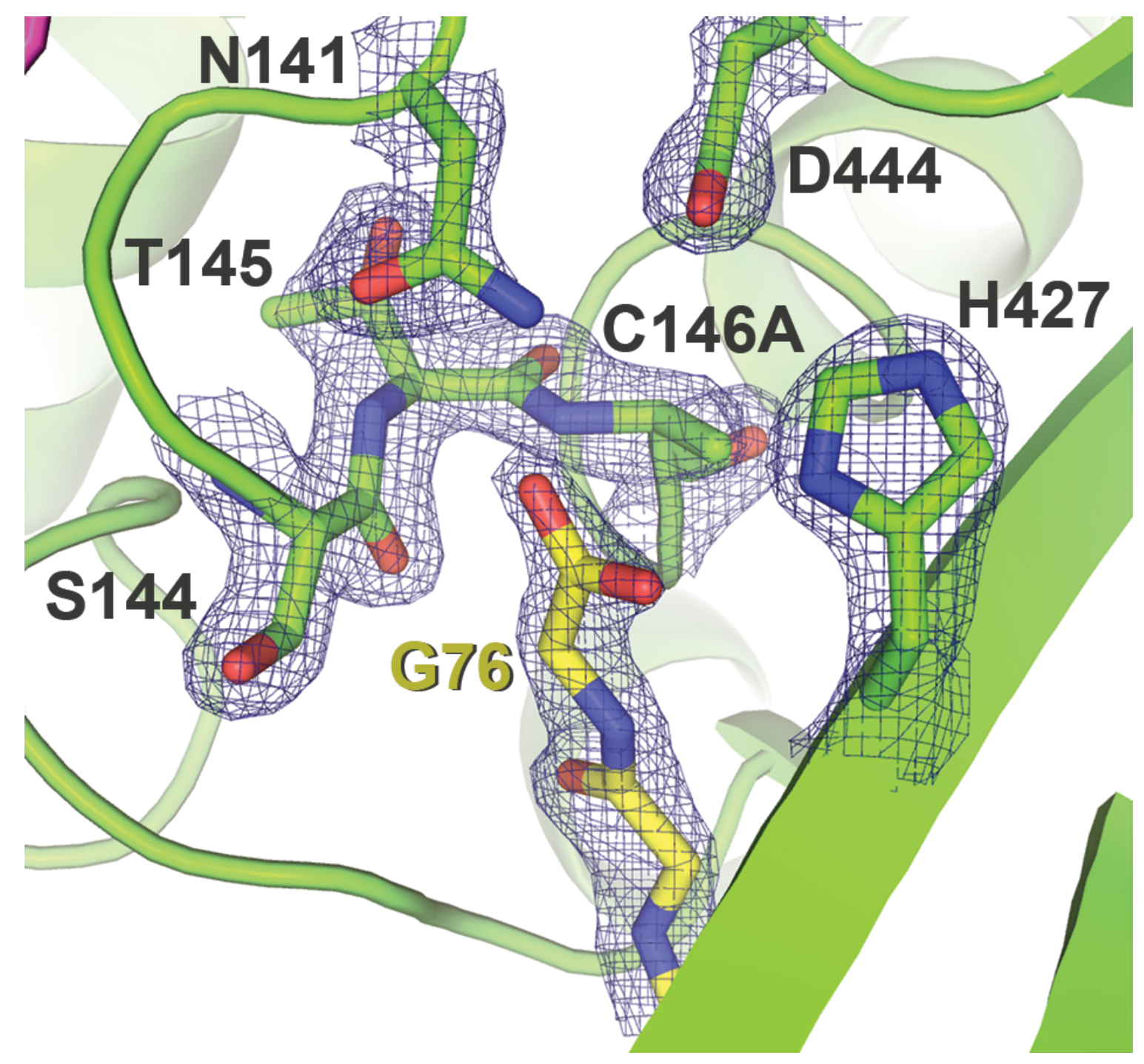

Figure S2: Active site of DUBm-Ubp8 ${ }^{\mathrm{C} 146 \mathrm{~A}}$ bound to monoubiquitin. Density is rendered from the $2 \mathrm{~F}_{\mathrm{o}}-\mathrm{F}_{\mathrm{c}}$ map and contoured to $2 \sigma$. 


\section{Figure S3}
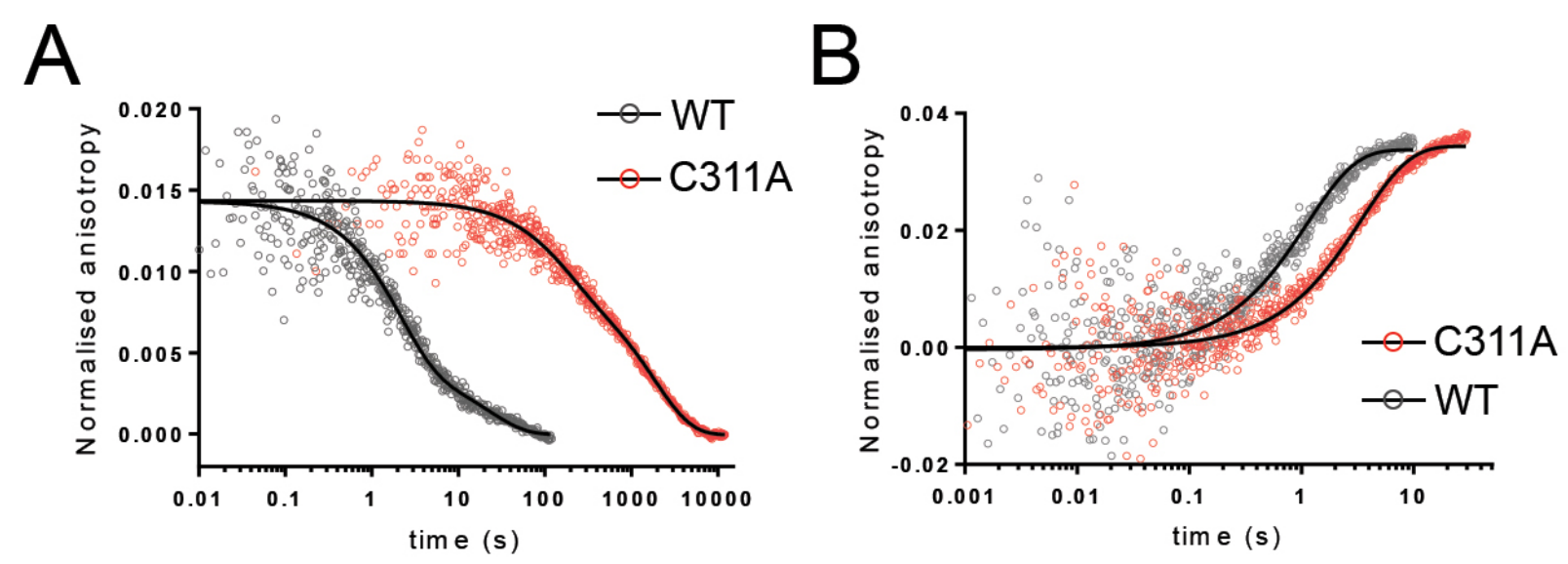

\begin{tabular}{|c|c|c|}
\hline & $\mathbf{K}_{\text {slow }}\left(\mathbf{s}^{-1}\right)$ & $\mathbf{K}_{\text {fast }}\left(\mathbf{s}^{-1}\right)$ \\
\hline USP4 $\mathbf{~ w t}$ & 0.053 & 0.55 \\
\hline USP4 CA & 0.00055 & 0.0063 \\
\hline
\end{tabular}

\begin{tabular}{|c|c|}
\hline & $\mathrm{K}_{\text {on }}\left(\mu \mathrm{M}^{-1} \mathbf{s}^{-1}\right)$ \\
\hline USP4 wt & 5.1 \\
\hline USP4 CA & 2.0 \\
\hline
\end{tabular}
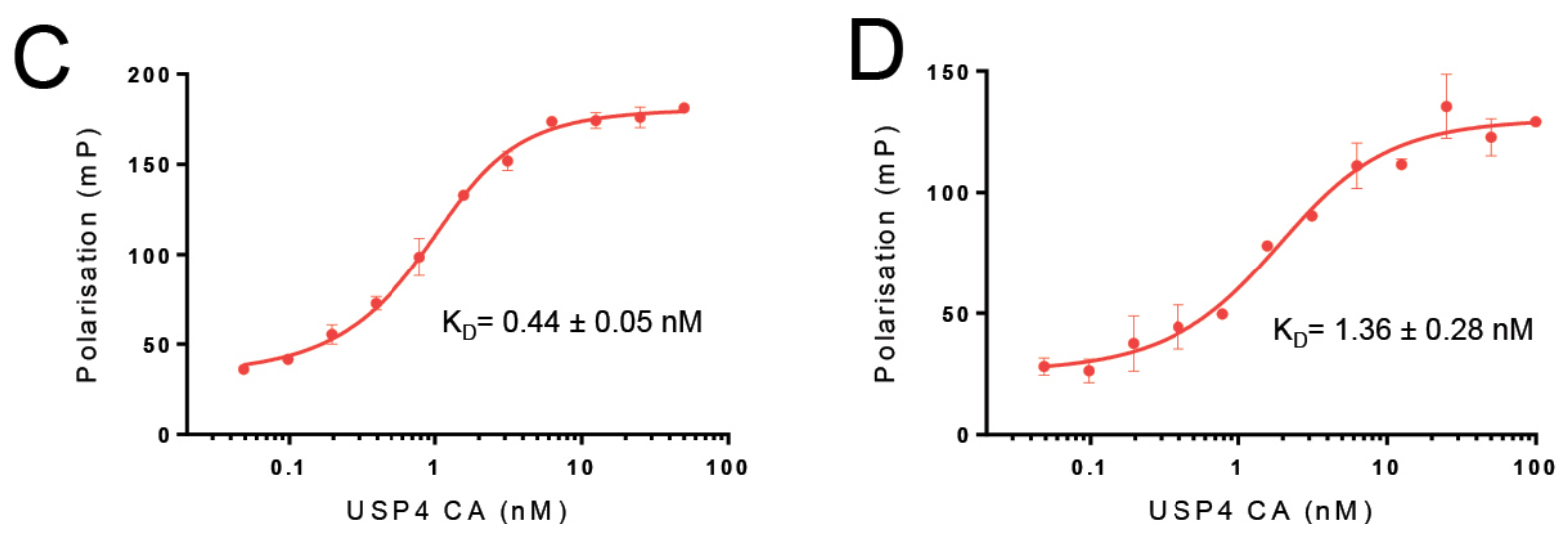

Figure S3: Binding kinetics of USP4 WT and C311A to ubiquitin substrates. (A) Dissociation kinetics of USP4 WT and C311A binding to TAMRA-monoubiquitin, measured by stopped flow fluorescence polarization. (B) Association kinetics for USP4 WT and C311A binding to TAMRA-monoubiquitin. Measured by stopped flow fluorescence polarization. (C) Equilibrium binding of USP4 C311A to TAMRA-ubiquitin conjugated to lysine. (D) Equilibrium binding of USP4 C311A to TAMRA-ubiquitin conjugated to an 18-mer peptide derived from SMAD4. 